\title{
Genes Involved in Neurodevelopment, Neuroplasticity and Major Depression: No Association for CACNA1C, CHRNA7 and MAPK1
}

\begin{abstract}
Marco Calabrò ${ }^{1}$, Laura Mandelli ${ }^{2}$, Concetta Crisafulli ${ }^{1}$, Soo-Jung Lee ${ }^{3}$, Tae-Youn Jun ${ }^{3}$, Sheng-Min Wang ${ }^{3}$, Ashwin A. Patkar, Prakash S. Masand ${ }^{5,6}$, Changsu Han ${ }^{7}$, Chi-Un Pae ${ }^{3,4,8}$, Alessandro Serretti ${ }^{2}$

${ }^{1}$ Department of Biomedical and Dental Sciences and Morphofunctional Imaging, University of Messina, Messina, ${ }^{2}$ Department of Biomedical and Neuromotor Sciences, University of Bologna, Bologna, Italy, ${ }^{3}$ Department of Psychiatry and ${ }^{8}$ Cell Death Disease Research Center, College of Medicine, The Catholic University of Korea, Seoul, Korea, ${ }^{4}$ Department of Psychiatry and Behavioural Sciences, Duke University Medical Center, Durham, NC, USA, ${ }^{5}$ Academic Medicine Education Institute, Duke-NUS Medical School, Singapore, Singapore, ${ }^{6}$ Global Medical Education, New York, NY, USA, ${ }^{7}$ Department of Psychiatry, Korea University College of Medicine, Seoul, Korea
\end{abstract}

\begin{abstract}
Objective: Genetics factors are likely to play a role in the risk, clinical presentation and treatment outcome in major depressive disorder (MDD). In this study, we investigated the role of three candidate genes for MDD; calcium voltagegated channel subunit alpha1 $\mathrm{C}(C A C N A 1 C)$, cholinergic receptor nicotinic alpha 7 subunit $(C H R N A 7)$, and mitogenactivated protein kinase 1 (MAPK1).

Methods: Two-hundred forty-two MDD patients and 326 healthy controls of Korean ancestry served as samples for the analyses. Thirty-nine single nucleotide polymorphisms (SNPS) within CACNA1C, CHRNA7, and MAPK1 genes were genotyped and subsequently tested for association with MDD (primary analysis) and other clinical features (symptoms' severity, age of onset, history of suicide attempt, treatment outcome) (secondary analyses). Single SNPs, haplotypes and epistatic analyses were performed.

Results: Single SNPs were not associated with disease risk and clinical features. However, a combination of alleles (haplotype) within MAPK1 was found associated with MDD-status. Secondary analyses detected a possible involvement of $C A C N A 1 C$ haplotype in resistance to antidepressant treatment.

Conclusion: These data suggest a role for MAPK1 and CACNA1C in MDD risk and treatment resistance, respectively. However, since many limitations characterize the analysis, the results must be considered with great caution and verified.
\end{abstract}

KEY WORDS: MAPK1; CACNA1C; CHRNAT; Major depressive disorder; Deep phenotyping.

\section{INTRODUCTION}

Major depressive disorder (MDD) is a severe psychopathological condition that has as main characteristic a severe alteration of mood and self-image, along with somatic and vegetative symptoms. ${ }^{1)}$ The high disabling potential of this condition, as well as the growing number of cases worldwide, has led MDD to be one of the most investigated psychiatric disorders. ${ }^{2)}$

There is evidence of a genetic liability for MDD, and

Received: November 29, 2017 / Accepted: December 4, 2017

Address for correspondence: Chi-Un Pae

Department of Psychiatry, The Catholic University of Korea,

Bucheon St. Mary's Hospital, 327 Sosa-ro, Wonmi-gu, Bucheon

14647, Korea

E-mail: pae@catholic.ac.kr

ORCID: https://orcid.org/0000-0003-1632-4248 several genes have been associated with the disease in case-control and wide-genome studies. Despite this, little is known about the specific effects of the genes (and their alterations) on disease risk and other clinical features. ${ }^{3,4)}$

Among several investigated genes, calcium voltage-gated channel subunit alpha1 C (CACNA1C), cholinergic receptor nicotinic alpha 7 subunit (CHRNAT), and mitogen-activated protein kinase 1 (MAPK1) have raised interest, since they encode for products involved in key nodes of several brain processes related to MDD. ${ }^{5-13)}$ To some extent, they are involved in dendritic development, neuronal survival, synaptic plasticity and memory/ learning. ${ }^{14-16)}$

CACNA1C encodes for an L-typed voltage-dependent calcium channel subunit contributing to channels that influence neuronal excitability. Its potential role in MDD

(ㄷ) This is an Open-Access article distributed under the terms of the Creative Commons Attribution Non-Commercial License (http://creativecommons.org/licenses/by-nc/4.0) which permits unrestricted non-commercial use, distribution, and reproduction in any medium, provided the original work is properly cited. 
was already investigated in literature. ${ }^{11-13)}$ MAPK1 encoded protein is implicated in a variety of biological processes and it has a critical role in synaptic and structural plasticity. MAPK1 is also involved in the initiation and progression of inflammatory processes, which are highly related to depressive states. ${ }^{5-7,17,18)}$ CHRNA7 encoded receptor is involved in cognition through interneuron modulation of dopamine and glutamate signaling. Evidence of its involvement in psychiatric disorders has also been reported, especially in antidepressant mechanisms. ${ }^{8,9)}$

In a previous case-control study, we detected an association of CACNA1C, MAPK1, and CHRNATgenes with bipolar disorder (BPD) risk. ${ }^{19)}$ Further, in previous studies we detected a possible involvement on CACNA1C in antidepressant response ${ }^{20)}$ and an association of MAPK1 with remission. ${ }^{17)}$ Given such evidence, here we further investigated these genes in MDD evaluating independent samples of patients and controls. In order to explore possible minor effects on specific disease characteristics, we also evaluated a potential impact on age of onset, history of suicide attempt, symptoms severity and treatment outcome.

\section{METHODS}

\section{Subjects}

Two-hundred and forty-two MDD patients according to the Diagnostic and Statistical Manual of Mental Disorders 4th edition (DSM-IV) criteria $^{21)}$ consecutively admitted to the Department of Psychiatry in Seoul St. Mary's Hospital for MDD treatment, and 326 healthy controls, were used as samples in this study. Recruitment details and exclusion criteria have been previously reported. ${ }^{19)}$ Briefly, patients were treated with paroxetine and/or venlafaxine and evaluated for antidepressant response approximately after six weeks of treatment. Controls were recruited among hospital staff and non-psychiatric hospital patients. In order to be considered controls they did not have to meet criteria for a major current psychiatric condition. Subjects with severe or unstable medical and/or neurological conditions, in treatment with a long-acting antipsychotic, with current or recent (past six months) comorbidity with alcohol and substance abuse disorders were not eligible for the study. All individuals were Koreans of Korean ascendants. The local ethical committee approved the study procedures and all the subjects were included after they had signed an in- formed consent.

\section{Evaluations}

The Mini-International Neuropsychiatric Interview $(\mathrm{MINI})^{21)}$ was employed for psychiatric evaluations. Demographic and clinical variables, including age at first illness episode (onset) and history of suicide attempt, were collected by clinical interview and review of clinical charts. Lifetime suicide attempt was defined as a non-fatal self-injurious behavior with intent to die. Depressive severity was evaluated by the Hamilton Rating Scale for Depression (HAMD). ${ }^{22)}$ Response to treatment, remission and resistance to treatments were defined according to Schosser et al. ${ }^{23)}$ Briefly, response to treatment is defined by $50 \%$ improvement of HAMD scores from baseline to endpoint; remission by a HAMD score of 7 or less at the endpoint; resistance by non-response to at least two adequate consecutive antidepressant trials. ${ }^{23)}$

\section{Genetic Analysis}

A total of 39 single nucleotide polymorphisms (SNPs) within CACNA1C, CHRNA7, and MAPK1 genes were tested for association with MDD and clinical features (Supplementary Table 1). Analyses were performed by independent investigators who were blind to the status of the subjects. Samples showing ambiguous alleles on repeated genotyping were discarded. For details about gene selection and genotypization please refer to Calabrò et al. ${ }^{19)}$

\section{Statistical Analysis}

Hardy-Weinberg equilibrium (HWE) and linkage disequilibrium (LD) were tested by Haploview 3.2 software for Windows. ${ }^{24)}$ Case-control analysis and genetic associations with clinical variables were analyzed using the IBM SPSS package for Windows ver. 23 (IBM Corp., Armonk, NY, USA). Haplotypes' analysis was performed in "R" environment (http://cran.r-project.org/), using the statistics package "haplo.score". SNPs were combined according to LD blocks. The sliding windows method was applied to narrow the block of SNPs analyzed for each LD Block. Further, we set the analyses to perform a minimum of 10,000 and a maximum of 50,000 simulations for each haplotype analyzed. The simulated $p$ value is reported together with the global $p$ value.

Finally, possible epistatic effects ${ }^{25)}$ were evaluated 
through multifactor dimensionality reduction test (MDR, http://www.multifactordimensionalityreduction.org/). Further details on the methods used can be found in Calabrò et al. ${ }^{19)}$ To control for multiple testing, the false discovery rate method (FDR ${ }^{26)}$ was employed to evaluate the significance of findings. Significance was considered for corrected $p<0.05$.

\section{RESULTS}

\section{Demographic Data}

Sociodemographic data of the sample under investigation are reported in Supplementary Table 1. No significant differences between cases and controls were observed in terms of age and gender (respectively $38.0 \%$ and $54.9 \%$ females, aged $43.6 \pm 15$ years and $45.4 \pm 13$ years). In patients, mean age at onset was 39.8 ( \pm 14 ) years. Fifty-four patients $(22.3 \%)$ had a positive history of suicide attempt. In our samples, all the SNPs analyzed were in HWE (Supplementary Table 2). Supplementary Figure 1 reports the results of $L D$ test.

\section{Risk of MDD}

In single SNP analyses, alleles and genotypes were not differentially distributed between cases and controls (all $p$ $<0.05$ ) (Supplementary Table 3). However, a block of al-

Table 1. Association between rs8136867-rs9610417 MAPK1 haplotypes with MDD*

\begin{tabular}{cccccc}
\hline rs8136867 & rs9610417 & Simulated $p$ & $p$ & Hap-score & Hap-freq \\
\hline g & $\mathrm{c}$ & 0.015 & 0.012 & -2.512 & 0.592 \\
$\mathrm{a}$ & $\mathrm{C}$ & 0.329 & 0.337 & 0.960 & 0.304 \\
$\mathrm{a}$ & $\mathrm{t}$ & 0.013 & 0.013 & 2.482 & 0.102 \\
\hline
\end{tabular}

MDD, major depressive disorder; Hap-score, haplotype score on phenotype; Hap-freq, haplotype frequency.

*Stat $=0.032$ and global $p=0.010$. leles in MAPK1 (rs8136867-rs9610417) was associated with MDD (global $p=0.010$ ). In particular, the most frequent GC haplotype was associated with control-status, while the less frequent AT haplotype was associated with MDD-status (Table 1). Tests for epistatic effects (MDR) did not provide a significant model for MDD risk (data not shown, available on request).

\section{Symptoms Severity, Age of Onset and History of Suicide Attempt}

Exploratory analyses did not provide evidence for any association between depressive severity, age of onset and suicide attempt history with the genetic variants considered in our study (Supplementary Table 4). By haplotypes analysis, trends of association with symptom severity at baseline (CHRNAT and CACNA1C) and discharge (CACNA1C) could be observed (Supplementary Table 5), but above the threshold of significance. Tests for epistasis (MDR) did not provide any significant model for these phenotypes.

\section{Treatment Outcomes (Response, Remission and Resistance to Treatment)}

Single SNPs were not significantly associated with measures of treatment outcome (all $p<0.05$ ). However, a four-SNPs haplotype within CACNA1C (rs880342rs11062196-rs2238062-rs3819536) was associated with treatment resistance (Table 2). Two SNPs (rs880342 and rs3819536) mainly contributed to treatment resistance, with the allelic CG combination being more frequent in the non-resistant group and the TA block being more common in the resistant group. However, the different distribution between the two groups was significant only when the central pair of SNPs (rs11062196 rs2238062) haplotype was GA. Tests for epistasis (MDR) did not provide a significant model for any of the investigated outcomes (response, remission and resistance).

Table 2. Association between 4 SNPs haplotypes in CACNA1C with treatment resistance in MDD patients*

\begin{tabular}{cccccccc}
\hline rs880342 & rs11062196 & rs2238062 & rs3819536 & Simulated $p$ & $p$ & Hap-score & Hap-freq \\
\hline c & $\mathrm{g}$ & $\mathrm{a}$ & $\mathrm{g}$ & 0.019 & 0.019 & -2.338 \\
$\mathrm{t}$ & $\mathrm{g}$ & $\mathrm{a}$ & $\mathrm{g}$ & 0.604 & 0.59 & -0.539 \\
$\mathrm{C}$ & $\mathrm{g}$ & $\mathrm{a}$ & $\mathrm{a}$ & 0.722 & 0.717 & 0.079 & 0.362 \\
$\mathrm{t}$ & $\mathrm{g}$ & $\mathrm{a}$ & $\mathrm{a}$ & 0.001 & 0.001 & 3.348 \\
\hline
\end{tabular}

MDD, major depressive disorder; Hap-score, haplotype score on phenotype; Hap-freq, haplotype frequency.

$*$ Stat $=0.004$ and global $p=0.012$. 


\section{DISCUSSION}

As a main finding, we found a block of alleles within MAPK1 (rs8136867-rs9610417 haplotype) associated with MDD risk. In particular, the GC combination (more frequent in controls) might be protective, while the opposite AT combination (more frequent in MDD cases) might increase disease risk. The two SNPs (both intronic) are about $5 \mathrm{~kb}$ from each other, and do not seem to fall on methylation points. However, according to the Human Splicing Finder (http://www.umd.be/HSF3/), an online prediction software, mutation on these sites may alter enough the sequence to broke/insert specific motifs and thus alter, quantitatively or qualitatively, $M A P K 1$ product. The presence of both alterations may increase this effect (as supported by their analysis as a block). In particular, the AT variant might produce the loss of a potential splice site and 3 enhancer motifs. Of note, one of the SNPs included in the haplotype, rs8136867, was previously associated with treatment efficacy in Caucasian MDD subjects. ${ }^{17)}$ However, we could not replicate the association with treatment outcome in our sample.

As regards treatment outcomes, extending our previous results on an independent sample evaluated for different SNPs within $C A C N A 1 C^{20)}$ in this study we identified a haplotype (rs880342-rs11062196-rs2238062-rs3819536) potentially associated with resistance to treatments, i.e. multiple non-response to adequate antidepressant treatment trials. Within this block, rs880342 and rs3819536 exerted a major influence on the treatment-resistant phenotype, with CG variant being protective while TA more frequent in the resistant patients group. These variations may cause some changes to the sequence, since they both alter a CG sequence with a possible impact on methylation mechanics. Further, an analysis with Human Splicing Finder for consensus motifs revealed that the TA haplotype loses 3 enhancer motifs and it produce a possible slice site and an additional silencer motif. As such, an effect of this haplotype on $C A C N A 1 C$ seems plausible. This data adds to our previous results linking $C A C N A 1 C$ alterations with antidepressant efficacy. ${ }^{20)}$

We did not find any significant epistatic model in this sample, suggesting that these different genes work independently from each other in MDD risk. This result slightly differs from that we obtained in BPD patients, ${ }^{19)}$ where SNPs in these different genes seems to have syner- gic effects (rs1016388 within CACNA1C, rs1514250, rs2337980, rs6494223, rs3826029 and rs4779565 within CHRNA7 and rs8136867 within MAPK1 were significantly associated with BPD).

In complex diseases, single genetic variants contribute to disease risk only marginally. Synergic effects between SNPs can generate slightly stronger signals. This may explain why we did not find association in single SNPs analysis, while we found seemingly positive results in haplotype analysis.

The small sample size and the high number of tests performed represent major limitations of our study, strongly preventing us from drawing reliable conclusions. Though previous convincing evidence suggesting MAPK1 in MDD, ${ }^{17,18)}$ its association with MDD in our Korean sample was small in terms of effect size and it should be therefore taken with caution. Ethnic differences prevent the generalization of the results to other populations. The genotyping methodology was not able to discriminate whether contiguous SNPs are located on the same chromosome (cis) or not (trans). Thus, haplotypes can only be inferred by a probabilistic approach.

In conclusion, the results of our study cautiously suggest an involvement of MAPK1 in MDD in Asians, though only considering a specific combination of alleles rather than single mutations. CACNA1C might influence treatment outcome. However, these data are very preliminary and need to be verified.

\section{- Acknowledgments}

This research was supported by a grant of the Korea Health Technology R\&D Project through the Korea Health Industry Development Institute (KHIDI), funded by the Ministry of Health \& Welfare, Republic of Korea (Grant Number : HC15C1405). All authors declare no personal, financial, or commercial conflicts of interest in relation with this study.

\section{Conflicts of Interest}

No potential conflict of interest relevant to this article was reported.

\section{SUPPLEMENTARY MATERIALS}

Supplementary data are available at https://doi.org/ 10.9758/cpn.2019.17.3.364. 


\section{REFERENCES}

1. Berrios GE. The psychopathology of affectivity: conceptual and historical aspects. Psychol Med 1985; 15:745-758.

2. Otte C, Gold SM, Penninx BW, Pariante CM, Etkin A, Fava M, et al. Major depressive disorder. Nat Rev Dis Primers 2016;2: 16065.

3. Serretti A. The present and future of precision medicine in psychiatry: focus on clinical psychopharmacology of antidepressants. Clin Psychopharmacol Neurosci 2018;16:1-6.

4. Zhao J, Wu X, Nie S, Gao X, Sun J, Li K, et al. Association of CDKN2B-AS1 rs1333049 with brain diseases: a case-control study and a meta-analysis. Clin Psychopharmacol Neurosci 2017;15:53-58.

5. Krishnan V, Nestler EJ. Linking molecules to mood: new insight into the biology of depression. Am I Psychiatry 2010; 167:1305-1320.

6. Vogelzangs N, Duivis HE, Beekman AT, Kluft C, Neuteboom J, Hoogendijk W, et al. Association of depressive disorders, depression characteristics and antidepressant medication with inflammation. Trans/ Psychiatry 2012;2:e79.

7. Zunszain PA, Hepgul N, Pariante CM. Inflammation and depression. Curr Top Behav Neurosci 2013;14:135-151.

8. Rabenstein RL, Caldarone BJ, Picciotto MR. The nicotinic antagonist mecamylamine has antidepressant-like effects in wild-type but not beta2- or alpha7-nicotinic acety/choline receptor subunit knockout mice. Psychopharmacology (Berl) 2006; 189:395-401.

9. Shytle RD, Silver AA, Lukas RJ, Newman MB, Sheehan DV, Sanberg PR. Nicotinic acety/choline receptors as targets for antidepressants. Mol Psychiatry 2002; 7:525-535.

10. Drago A, Crisafulli C, Sidoti A, Serretti A. The molecular interaction between the glutamatergic, noradrenergic, dopaminergic and serotoninergic systems informs a detailed genetic perspective on depressive phenotypes. Prog Neurobiol 2011; 94:418-460.

11. Dedic N, Pöhlmann ML, Richter JS, Mehta D, Czamara D, Metzger MW, et al. Cross-disorder risk gene CACNA1C differentially modulates susceptibility to psychiatric disorders during development and adulthood. Mol Psychiatry 2018;23:533-543.

12. Rao $\mathrm{S}$, Yao $\mathrm{Y}$, Zheng $\mathrm{C}$, Ryan J, Mao $\mathrm{C}$, Zhang F, et al. Common variants in CACNA1C and MDD susceptibility: A comprehensive meta-analysis. Am J Med Genet B Neuropsychiatr Genet 2016;171:896-903.

13. He K, An Z, Wang Q, Li T, Li Z, Chen J, et al. CACNA1C, schizophrenia and major depressive disorder in the Han Chinese population. Br J Psychiatry 2014;204:36-39.

14. Berger SM, Bartsch D. The role of L-type voltage-gated cal- cium channels Cav1.2 and Cav1.3 in normal and pathological brain function. Cell Tissue Res 2014;357:463-476.

15. Levin ED. $\alpha 7$-Nicotinic receptors and cognition. Curr Drug Targets 2012;13:602-606.

16. Hetman M, Gozdz A. Role of extracellular signal regulated kinases 1 and 2 in neuronal survival. EurJ Biochem 2004;271: 2050-2055.

17. Calati R, Crisafulli C, Balestri M, Serretti A, Spina E, Calabrò $\mathrm{M}$, et al. Evaluation of the role of MAPK1 and CREB1 polymorphisms on treatment resistance, response and remission in mood disorder patients. Prog Neuropsychopharmacol Biol Psychiatry 2013;44:271-278.

18. García-Fuster MJ, Díez-Alarcia R, Ferrer-Alcón M, La Harpe R, Meana JJ, García-Sevilla JA. FADD adaptor and PEA-15/ ERK1/2 partners in major depression and schizophrenia postmortem brains: basal contents and effects of psychotropic treatments. Neuroscience 2014;277:541-551.

19. Calabrò M, Mandelli L, Crisafulli C, Sidoti A, Jun TY, Lee SJ, et al. Genes involved in neurodevelopment, neuroplasticity, and bipolar disorder: CACNA1C, CHRNA1, and MAPK1. Neuropsychobiology 2016;74:159-168.

20. Cocchi E, Fabbri C, Han C, Lee SJ, Patkar AA, Masand PS, et al. Genome-wide association study of antidepressant response: involvement of the inorganic cation transmembrane transporter activity pathway. BMC Psychiatry 2016;16:106.

21. Sheehan DV, Lecrubier $Y$, Sheehan $K H$, Amorim $P$, Janavs J, Weiller E, et al. The Mini-International Neuropsychiatric Interview (M.I.N.I.): the development and validation of a structured diagnostic psychiatric interview for DSM-IV and ICD-10. I Clin Psychiatry 1998;59 Suppl 20:22-33;quiz 34-57.

22. Zimmerman M, Martinez JH, Young D, Chelminski I, Dalrymple K. Severity classification on the Hamilton Depression Rating Scale. J Affect Disord 2013;150:384-388.

23. Schosser A, Serretti A, Souery D, Mendlewicz J, Zohar J, Montgomery S, et al. European Group for the Study of Resistant Depression (GSRD)--where have we gone so far: review of clinical and genetic findings. Eur Neuropsychopharmacol 2012;22:453-468.

24. Barrett JC, Fry B, Maller J, Daly MJ. Haploview: analysis and visualization of $L D$ and haplotype maps. Bioinformatics 2005;21:263-265.

25. Rosand J. Epistasis is coming: are we ready? Stroke 2005;36: 1879-1880.

26. Benjamini Y, Drai D, Elmer G, Kafkafi N, Golani I. Controlling the false discovery rate in behavior genetics research. Behav Brain Res 2001;125:279-284. 